\title{
Reply: Timeliness, risk communication and patient preferences for investigations or referral
}

\author{
Georgios Lyratzopoulos ${ }^{*}, 1$, Gary A Abel ${ }^{1}$, Sean McPhail ${ }^{2}$, Richard D Neal ${ }^{3}$ and Gregory P Rubin ${ }^{4}$ \\ ${ }^{1}$ Cambridge Centre for Health Services Research, Department of Public Health and Primary Care, University of Cambridge, \\ Cambridge CB2 OSR, UK; ${ }^{2}$ National Cancer Intelligence Network (NCIN), 18th Floor, Portland House, Bressenden Place, \\ London SW1E 5RS, UK; ${ }^{3}$ North Wales Centre for Primary Care Research, North Wales Clinical School, College of Health and \\ Behavioural Sciences, Bangor University, Gwenfro Units 4-8, Wrexham Technology Park, Wrexham LL13 7YP, UK and ${ }^{4}$ Wolfson \\ Research Institute, School of Medicine and Health, University of Durham, Queen's Campus, University Boulevard, Stockton-on- \\ Tees TS17 6BH, UK
}

Sir,

We thank the authors for their reflections (Irving and Holden, 2013). As they acknowledge, we did not endorse the notion that optimal care means correctly identifying and referring a patient with cancer during the first consultation (Lyratzopoulos et al, 2013). We question whether this notion is indeed 'widely held'. In the United Kingdom, policy makers and cancer charities often use a binary measure of promptness of cancer diagnosis comprising one or two (compared with three or more) consultations, for example, in public reporting of NHS patient experience surveys (Department of Health, 2012). This is also the measure mainly profiled in our paper. The main conclusions of our paper were that the number of pre-referral consultations is a good surrogate marker of the primary care interval; and that there is large variation in respect of both measures between patients with different cancers.

We would agree in principle that some repeat consultations may be appropriate, for example, within an 'active monitoring' (or 'safety-netting') framework. The challenge for the primary care physician is to identify the earliest point at which the possibility of a cancer diagnosis would justify investigation or referral. Such decisions are informed by knowledge of cancer risk for given symptoms and by clinical practice guidelines (NICE, 2005; Hamilton, 2009). However, any decision to delay investigation or referral as part of 'active monitoring' must take into account patient preferences and the potential risk of disease progression. Recent UK research indicates that patients express a strong preference for investigation of symptoms likely to be due to cancer, even when the positive predictive value of such symptoms is known to be low (Hollinghurst et al, 2013). Therefore, doctors need to ensure that whenever deferral of investigation or referral is being considered, the associated uncertainties and risks are communicated and shared with patients. We note the authors' work on the time efficiency principle, but because biological processes of disease progression vary between medical conditions, the importance of timeliness of diagnosis and treatment may well be different in the context of cancer (Chen et al, 2008).

The authors are correct in their assertion that cancer presentations in primary care are relatively rare, but we would wish to correct the illustration regarding multiple myeloma and stomach cancer provided by the authors, who have overinterpreted data in our paper. As we have previously alluded to, data from the National Audit of Cancer Diagnosis in Primary Care largely reflect the cancer 'case-mix' observed in population-based incidence statistics but cannot be used to estimate incidence, not least because data collection periods varied from 6 to 12 months between participating practices. Using Office for National Statistics data, the incidence per practice of multiple myeloma and stomach cancer is in the order of 1 case every 2 years and 1 case every 18 months, respectively.

\section{REFERENCES}

Chen Z, King W, Pearcey R, Kerba M, Mackillop WJ (2008) The relationship between waiting time for radiotherapy and clinical outcomes: a systematic review of the literature. Radiother Oncol 87(1): $3-16$

Department of Health (2012) Cancer Patient Experience Survey 2011/12 https://www.gov.uk/government/publications/second-national-cancerpatient-experience-survey (last accessed March 2013). 
Hamilton W (2009) The CAPER studies: five case-control studies aimed at identifying and quantifying the risk of cancer in symptomatic primary care patients. Br J Cancer 101(Suppl 2): S80-S86.

Hollinghurst S, Banks J, Peters T, Bigwood L, Walter F, Hamilton W. (2013) Patient views on being referred for diagnostic testing for cancer-a vignette based survey. Oral presentation, Society for Academic Primary Care-South West, Annual Research Meeting, Southampton.
Irving G, Holden J (2013) Measures of promptness of cancer diagnosis in primary care. Br J Cancer 108(10): 2186.

Lyratzopoulos G, Abel GA, McPhail S, Neal RD, Rubin GP (2013) Measures of promptness of cancer diagnosis in primary care: secondary analysis of national audit data on patients with 18 common and rarer cancers. Br J Cancer 108(3): 686-690.

NICE (National Institute for Health and Clinical Excellence) (2005) Clinical Guideline 27 Referral for suspected cancer: NICE guideline http://www.nice. org.uk/nicemedia/pdf/CG027quickrefguide.pdf (last accessed March 2013). 